PROCEEDINGS OF THE

AMERICAN MATHEMATICAL SOCIETY

Volume 135, Number 11, November 2007, Pages 3649-3658

S 0002-9939(07)08927-7

Article electronically published on August 7, 2007

\title{
MULTIPLE NONTRIVIAL SOLUTIONS FOR NONLINEAR EIGENVALUE PROBLEMS
}

\author{
D. MOTREANU, V. V. MOTREANU, AND N. S. PAPAGEORGIOU
}

(Communicated by David S. Tartakoff)

\begin{abstract}
In this paper we study a nonlinear eigenvalue problem driven by the $p$-Laplacian. Assuming for the right-hand side nonlinearity only unilateral and sign conditions near zero, we prove the existence of three nontrivial solutions, two of which have constant sign (one is strictly positive and the other is strictly negative), while the third one belongs to the order interval formed by the two opposite constant sign solutions. The approach relies on a combination of variational and minimization methods coupled with the construction of upper-lower solutions. The framework of the paper incorporates problems with concave-convex nonlinearities.
\end{abstract}

\section{InTROduCtion AND STATEMENT OF MAin RESUlt}

In this paper we study the existence of multiple solutions of the following nonlinear eigenvalue problem: find $u \in W_{0}^{1, p}(\Omega) \backslash\{0\}$ and $\lambda>0$ such that

$$
\begin{cases}-\Delta_{p} u=f(x, u, \lambda) & \text { in } \Omega \\ u=0 & \text { on } \partial \Omega\end{cases}
$$

where $\Omega \subset \mathbb{R}^{N}$ is a bounded domain with a $C^{2}$-boundary $\partial \Omega$ and $1<p<+\infty$. In problem (1.1) we have the negative $p$-Laplacian operator $-\Delta_{p}: W_{0}^{1, p}(\Omega) \rightarrow$ $W^{-1, p^{\prime}}(\Omega)\left(\frac{1}{p}+\frac{1}{p^{\prime}}=1\right)$ acting as

$$
\left\langle-\Delta_{p} u, v\right\rangle=\int_{\Omega}\|D u(x)\|^{p-2}(D u(x), D v(x))_{\mathbb{R}^{N}} d x \text { for all } u, v \in W_{0}^{1, p}(\Omega) .
$$

Our aim is to study the multiplicity of solutions for all values of the parameter $\lambda$ in an interval $\left(0, \lambda_{0}\right)$, with $\lambda_{0}>0$. In the past this problem was examined primarily in the context of semilinear problems, i.e., for $p=2$. In this respect we cite the work of Ambrosetti-Brezis-Cerami 1 considering a concave-convex nonlinearity $f(x, s, \lambda)=\lambda|s|^{q-2} s+|s|^{r-2} s$, with $1<q<2<r<2^{*}$, and the one of Jin 8 . where $f(x, s, \lambda)$ is Hölder continuous with respect to $(x, s) \in \bar{\Omega} \times \mathbb{R}$ for every fixed $\lambda$. Concerning the nonlinear eigenvalue problems involving the $p$-Laplacian, we quote Garcia Azorero-Peral-Manfredi [6] focusing on the case where the potential

Received by the editors August 1, 2006 and, in revised form, September 6, 2006.

2000 Mathematics Subject Classification. Primary 35J60; Secondary 35J70.

Key words and phrases. Nonlinear eigenvalue problem, $p$-Laplacian, principal and second eigenvalue, upper-lower solution, truncation, strong maximum principle, second deformation lemma.

(C)2007 American Mathematical Society 
in problem (1.1) is given by $f(x, s, \lambda)=\lambda|s|^{q-2} s+|s|^{r-2} s$ with $1<q<p<r<p^{*}$, where

$$
p^{*}=\left\{\begin{array}{cl}
\frac{N p}{N-p} & \text { if } N>p \\
+\infty & \text { otherwise. }
\end{array}\right.
$$

We also mention the work of Carl-Perera [3], treating the multiplicity of solutions of problem (1.1) without the parameter $\lambda$, namely $f(x, s)=\alpha\left(s^{+}\right)^{p-1}-$ $\beta\left(s^{-}\right)^{p-1}+g(x, s)$, with $(\alpha, \beta) \in \mathbb{R}^{2}$ above the curve of the Fučik spectrum passing through $\left(\lambda_{2}, \lambda_{2}\right)\left(\lambda_{2}\right.$ denotes the second eigenvalue of $\left.\left(-\Delta_{p}, W_{0}^{1, p}(\Omega)\right)\right)$ and where $\lim _{s \rightarrow 0} \frac{g(x, s)}{\left.s\right|^{p-1}}=0$ uniformly in $x$.

Our hypotheses on the nonlinearity $f(x, s, \lambda)$ in problem (1.1) are the following:

$\mathrm{H}(f) f: \Omega \times \mathbb{R} \times(0, \bar{\lambda}) \rightarrow \mathbb{R}$, with $\bar{\lambda}>0$, is a function such that $f(x, 0, \lambda)=0$ a.e. on $\Omega$ whenever $\lambda \in(0, \bar{\lambda})$, and

(i) for all $\lambda \in(0, \bar{\lambda}), f(\cdot, \cdot, \lambda)$ is Carathéodory (that is, $f(\cdot, s, \lambda)$ is measurable for all $s \in \mathbb{R}$ and $f(x, \cdot, \lambda)$ is continuous for almost all $x \in \Omega$ );

(ii) there are constants $c>0, r>p-1$ and functions $a(\cdot, \lambda) \in L^{\infty}(\Omega)_{+}$ $(\lambda \in(0, \bar{\lambda}))$ with $\|a(\cdot, \lambda)\|_{\infty} \rightarrow 0$ as $\lambda \downarrow 0$ such that

$|f(x, s, \lambda)| \leq a(x, \lambda)+c|s|^{r}$ for a.a. $x \in \Omega$ and all $(s, \lambda) \in \mathbb{R} \times(0, \bar{\lambda})$;

(iii) for all $\lambda \in(0, \bar{\lambda})$ there exists $\mu>\lambda_{2}$ for which we have

$$
\mu<\liminf _{s \rightarrow 0} \frac{f(x, s, \lambda)}{|s|^{p-2} s} \text { uniformly for a.a. } x \in \Omega ;
$$

(iv) there exists a constant $b>0$ such that for almost all $x \in \Omega$, all $\lambda \in(0, \bar{\lambda})$ and all $s \in \mathbb{R}$ with $|s|<b$ we have $f(x, s, \lambda) s \geq 0$.

In order to formulate our main result, we recall that in the Banach space $C_{0}^{1}(\bar{\Omega})=$ $\left\{u \in C^{1}(\bar{\Omega}): u(x)=0, \forall x \in \partial \Omega\right\}$ the interior of the positive cone $C_{0}^{1}(\bar{\Omega})_{+}=\{u \in$ $\left.C_{0}^{1}(\bar{\Omega}): u(x) \geq 0, \forall x \in \Omega\right\}$ is given by

$$
\operatorname{int}\left(C_{0}^{1}(\bar{\Omega})_{+}\right)=\left\{u \in C_{0}^{1}(\bar{\Omega}): u(x)>0, \forall x \in \Omega \text {, and } \frac{\partial u}{\partial n}(x)<0, \forall x \in \partial \Omega\right\},
$$

where $n=n(x)$ is the outer unit normal at $x \in \partial \Omega$. We now state our main result on the existence of multiple nontrivial solutions to problem (1.1).

Theorem 1.1. Under hypotheses $\mathrm{H}(f)$, there is a number $\lambda_{0} \in(0, \bar{\lambda})$ such that for all $\lambda \in\left(0, \lambda_{0}\right)$ problem (1.1) has at least three distinct nontrivial solutions $u_{0}=u_{0}(\lambda) \in \operatorname{int}\left(C_{0}^{1}(\bar{\Omega})_{+}\right), v_{0}=v_{0}(\lambda) \in-\operatorname{int}\left(C_{0}^{1}(\bar{\Omega})_{+}\right)$and $w_{0}=w_{0}(\lambda) \in C_{0}^{1}(\bar{\Omega})$ satisfying $-b<v_{0}(x) \leq w_{0}(x) \leq u_{0}(x)<b$ for all $x \in \Omega$ and with negative energies, that is,

$$
\frac{1}{p}\|D u\|_{p}^{p}-\int_{\Omega} \int_{0}^{u(x)} f(x, s, \lambda) d s d x<0 \text { for } u \in\left\{u_{0}, v_{0}, w_{0}\right\} .
$$

The next example illustrates that large classes of nonlinear eigenvalue problems, among which those with concave-convex potentials, fit in our setting. For the sake of simplicity we drop the $x$ dependence for the potential $f$ in the right-hand side of equation (1.1).

Example 1.2. The situation treated in [6], including the concave-convex case of [1], is covered by our hypotheses. More generally, without assuming a subcritical growth, the function $f: \mathbb{R} \times(0,+\infty) \rightarrow \mathbb{R}$ given by

$$
f(s, \lambda)=c_{1} \lambda|s|^{q-2} s+c_{2}|s|^{r-2} s \text { for all }(s, \lambda) \in \mathbb{R} \times(0,+\infty),
$$


with $c_{1}, c_{2}>0$ and $1<q<p<r<+\infty$, satisfies hypotheses $\mathrm{H}(f)$. Indeed, (i), (iii), (iv) (with any $b>0$ ) are obviously verified, while (ii) holds since by Young inequality we have

$$
|f(s, \lambda)| \leq c_{1} \frac{r-q}{r-1} \lambda^{\frac{r-1}{r-q}}+\left(c_{1} \frac{q-1}{r-1}+c_{2}\right)|s|^{r-1} \text { for all }(s, \lambda) \in \mathbb{R} \times(0,+\infty) .
$$

Further examples of functions $f: \mathbb{R} \times(0,+\infty) \rightarrow \mathbb{R}$ verifying assumptions $\mathrm{H}(f)$ are

$$
f(s, \lambda)= \begin{cases}\lambda\left(|s|^{q-2} s+|s|^{r-2} s\right) & \text { if }|s| \leq 1 \\ 2 \lambda|s|^{\ell-2} s+s|s|^{t-3} \ln |s| & \text { if }|s|>1\end{cases}
$$

where $1<\min \{q, r\}<p, \max \{\ell, p\}<t$, and

$$
f(s, \lambda)= \begin{cases}\lambda\left(\max \left\{s^{q-1}, s^{r-1}\right\}+\ln \left(1+s^{t-1}\right)\right) & \text { if } s \geq 0 \\ \lambda\left(|s|^{q-2} s+\arctan s\right) & \text { if } s<0\end{cases}
$$

where $1<q<r, q<p, 1<t$. Notice that in the last example the function $f$ is not odd with respect to $s$. In all these cases, Theorem 1.1 can be applied to the corresponding problems (1.1).

Commenting on our hypotheses, it is seen from $\mathrm{H}(f)$ that we do not assume symmetries, subcritical growth or asymptotic conditions at infinity. We only impose that the function $f(\cdot, \cdot, \lambda)$ is Carathéodory, satisfies an arbitrary polynomial growth, a unilateral condition near zero related to the second eigenvalue $\lambda_{2}$ of $\left(-\Delta_{p}, W_{0}^{1, p}(\Omega)\right)$ and a sign condition near zero. We remark that the sign condition (in assumption $\mathrm{H}(f)(\mathrm{iv})$ ) cannot be derived from the unilateral condition (in $\mathrm{H}(f)(\mathrm{iii}))$ because the constant $b$ in $\mathrm{H}(f)(\mathrm{iv})$ must be independent on $\lambda$.

Our main result stated as Theorem 1.1 provides two opposite constant sign solutions uniformly bounded by the constant $b$ and a third nontrivial solution belonging to their order interval. We note that the result cannot be repeatedly used to yield new solutions by taking the constant $b$ in $\mathrm{H}(f)$ (iv) smaller than the uniform norm of the already found solutions because they depend on $\lambda$. Theorem 1.1 complements the works in [1, [3], 6], 8] (see also Example 1.2). Actually, it is an extension of Theorem 1 in 8 ] from semilinear problems to those driven by the $p$-Laplacian, except for a slightly different sign condition near zero. Specifically, in addition to the extension from $p=2$ to any $1<p<+\infty$, we drop the requirement on $f(\cdot, \cdot, \lambda)$ to be Hölder continuous on $\bar{\Omega} \times \mathbb{R}$, provide additional information in the conclusion and use an argument different from the one in 8 based on the pseudo-gradient vector field.

Concerning the method of proof, it is worth pointing out that here we cannot consider the usual Euler functional associated to problem (1.1), that is,

$$
\varphi(u, \lambda)=\frac{1}{p}\|D u\|_{p}^{p}-\int_{\Omega} \int_{0}^{u(x)} f(x, s, \lambda) d s d x
$$

whenever $u \in W_{0}^{1, p}(\Omega)$ and $\lambda \in(0, \bar{\lambda})$, due to the general growth condition in $\mathrm{H}(f)(\mathrm{ii})$, so the common variational method seeking the weak solutions of (1.1) as the critical points of $\varphi(\cdot, \lambda)$ is not applicable. Our approach combines variational, truncation and minimization techniques with construction of upper-lower solutions. First, in Proposition 3.1 we find an upper bound $\lambda_{0}$ of $\lambda$ such that for any $\lambda \in\left(0, \lambda_{0}\right)$ there exist two solutions $u_{0}=u_{0}(\lambda), v_{0}=v_{0}(\lambda)$ of problem (1.1) having opposite constant sign. These solutions arise as global minimizers for some functionals constructed by means of a positive upper solution and a negative 
lower solution. Then, through suitable variational techniques we obtain another nontrivial solution $w_{0}=w_{0}(\lambda)$ of (1.1) belonging to the order interval determined by $v_{0}, u_{0}$ with $w_{0} \neq u_{0}$ and $w_{0} \neq v_{0}$. The fact that $w_{0}$ is nontrivial is deduced by applying the second deformation lemma.

The rest of the paper is organized as follows. Section 2 recalls an essential mathematical background needed in the sequel. Section 3 sets forth the construction of two solutions of opposite constant sign. Section 4 contains the proof of Theorem 1.1.

\section{Mathematical BACKGRound}

In this section we briefly recall basic mathematical tools which will be used in the analysis of problem (1.1). Let us start with some properties regarding the spectrum of the negative $p$-Laplacian with Dirichlet boundary conditions on $\Omega$. We consider the nonlinear eigenvalue problem of $-\Delta_{p}$ on $W_{0}^{1, p}(\Omega)$ : find $u \in W_{0}^{1, p}(\Omega) \backslash\{0\}$ and $\lambda>0$ such that

$$
\begin{cases}-\Delta_{p} u=\lambda|u|^{p-2} u & \text { in } \Omega, \\ u=0 & \text { on } \partial \Omega .\end{cases}
$$

The first eigenvalue for (2.1), denoted by $\lambda_{1}$, is variationally characterized as

$$
\lambda_{1}=\min \left\{\frac{\|D u\|_{p}^{p}}{\|u\|_{p}^{p}}: u \in W_{0}^{1, p}(\Omega), u \neq 0\right\},
$$

and the minimum is realized at an eigenfunction $u_{1}$ which is taken to satisfy $\left\|u_{1}\right\|_{p}=$ 1. By using the strong maximum principle (see [12]), we may assume that $u_{1} \in$ $\operatorname{int}\left(C_{0}^{1}(\bar{\Omega})_{+}\right)$.

Taking into account that $\lambda_{1}$ is isolated, the second eigenvalue $\lambda_{2}$ of $-\Delta_{p}$ on $W_{0}^{1, p}(\Omega)$ satisfies

$$
\lambda_{2}=\inf \left\{\lambda: \lambda \text { is an eigenvalue of }\left(-\Delta_{p}, W_{0}^{1, p}(\Omega)\right), \lambda>\lambda_{1}\right\}>\lambda_{1}
$$

(see Anane-Tsouli [2]). A variational expression for $\lambda_{2}$ was given by Cuesta-de Figueiredo-Gossez [4] as follows:

$$
\lambda_{2}=\inf _{\gamma \in \Gamma_{0}} \max _{u \in \gamma([-1,1])}\|D u\|_{p}^{p},
$$

where

$$
\Gamma_{0}=\left\{\gamma \in C([-1,1], S): \gamma(-1)=-u_{1}, \gamma(1)=u_{1}\right\}
$$

$S=W_{0}^{1, p}(\Omega) \cap \partial B_{1}^{L^{p}(\Omega)}$ and $\partial B_{1}^{L^{p}(\Omega)}=\left\{u \in L^{p}(\Omega):\|u\|_{p}=1\right\}$.

Given $\varphi \in C^{1}(X)$ on a Banach space $X$ and $c \in \mathbb{R}$, we set $\varphi^{c}=\{u \in X$ : $\varphi(u) \leq c\}$ and $K_{c}(\varphi)=\left\{u \in X: \varphi^{\prime}(u)=0, \varphi(u)=c\right\}$. We recall that $\varphi$ satisfies the Palais-Smale condition at level $c \in \mathbb{R}$ (the $\mathrm{PS}_{c}$-condition for short) if any sequence $\left\{u_{n}\right\} \subset X$ verifying $\varphi\left(u_{n}\right) \rightarrow c$ and $\varphi^{\prime}\left(u_{n}\right) \rightarrow 0$ in $X^{*}$ as $n \rightarrow \infty$ has a strongly convergent subsequence. We refer to [10] for the necessary mathematical background concerning the critical point theory. For later use we also mention the second deformation lemma (see, e.g., [7, p. 366]).

Lemma 2.1. If $\varphi \in C^{1}(X),-\infty<a<b \leq+\infty, \varphi$ satisfies the $\mathrm{PS}_{c}$-condition for every $c \in[a, b), \varphi$ has no critical values in the open interval $(a, b)$ and $\varphi^{-1}(a)$ contains at most a finite number of critical points of $\varphi$, then there exists a continuous 


$$
\begin{aligned}
& \text { mapping } \eta:[0,1] \times\left(\varphi^{b} \backslash K_{b}(\varphi)\right) \rightarrow \varphi^{b} \text { such that } \\
& \qquad \begin{array}{c}
\varphi(\eta(t, u)) \leq \varphi(\eta(s, u)) \text { for all } t, s \in[0,1], s \leq t \text { and all } u \in \varphi^{b} \backslash K_{b}(\varphi), \\
\eta\left(1, \varphi^{b} \backslash K_{b}(\varphi)\right) \subset \varphi^{a}, \eta(0, \cdot)=\left.\mathrm{id}\right|_{\varphi^{b} \backslash K_{b}(\varphi)} \\
\eta(t, u)=u \text { for all }(t, u) \in[0,1] \times \varphi^{a} .
\end{array}
\end{aligned}
$$

\section{Constant sign solutions through local minima}

We start by generating two solutions of problem (1.1) having constant sign.

Proposition 3.1. Assume $\mathrm{H}(f)(\mathrm{i})$, (ii), (iv) and a weaker form of $\mathrm{H}(f)(\mathrm{iii})$ : for all $\lambda \in(0, \bar{\lambda})$ there exists $\mu>\lambda_{1}$ for which we have

$$
\mu<\liminf _{s \rightarrow 0} \frac{f(x, s, \lambda)}{|s|^{p-2} s} \quad \text { uniformly for a.a. } x \in \Omega .
$$

Then there is a number $\lambda_{0} \in(0, \bar{\lambda})$ such that if $\lambda \in\left(0, \lambda_{0}\right)$ problem (1.1) has a solution $u_{0}=u_{0}(\lambda) \in \operatorname{int}\left(C_{0}^{1}(\bar{\Omega})_{+}\right)$and a solution $v_{0}=v_{0}(\lambda) \in-\operatorname{int}\left(C_{0}^{1}(\bar{\Omega})_{+}\right)$ satisfying $\left\|u_{0}\right\|_{\infty}<b,\left\|v_{0}\right\|_{\infty}<b$ and with negative energies.

Proof. It is well known that there exists $e \in \operatorname{int}\left(C_{0}^{1}(\bar{\Omega})_{+}\right)$satisfying $-\Delta_{p} e=1$.

We claim that there is $\lambda_{0} \in(0, \bar{\lambda})$ with the property that if $\lambda \in\left(0, \lambda_{0}\right)$ we can choose $\xi_{0}=\xi_{0}(\lambda) \in\left(0, \frac{b}{\|e\|_{\infty}}\right)$ such that

$$
c\left(\xi_{0}\|e\|_{\infty}\right)^{r}+\|a(\cdot, \lambda)\|_{\infty}<\xi_{0}^{p-1},
$$

with $c>0$ and $a(\cdot, \lambda)$ as in $\mathrm{H}(f)($ ii). Arguing by contradiction, suppose that there is a sequence $\lambda_{n} \downarrow 0$ as $n \rightarrow \infty$ such that

$$
c\left(\xi\|e\|_{\infty}\right)^{r}+\left\|a\left(\cdot, \lambda_{n}\right)\right\|_{\infty} \geq \xi^{p-1} \text { for all } n \in \mathbb{N} \text { and } \xi \in\left(0, \frac{b}{\|e\|_{\infty}}\right) .
$$

Letting $n \rightarrow \infty$ we get $c\|e\|_{\infty}^{r} \xi^{r-p+1} \geq 1$ for all $\xi \in\left(0, \frac{b}{\|e\|_{\infty}}\right)$ because, by hypothesis $\mathrm{H}(f)$ (ii), we have $\|a(\cdot, \lambda)\|_{\infty} \rightarrow 0$ as $\lambda \downarrow 0$. Since $r>p-1$, we arrive at a contradiction as $\xi \downarrow 0$. Therefore (3.1) holds true.

Fix $\lambda \in\left(0, \lambda_{0}\right)$ and set $\bar{u}=\xi_{0} e \in \operatorname{int}\left(C_{0}^{1}(\bar{\Omega})_{+}\right)$, with $\xi_{0} \in\left(0, \frac{b}{\|e\|_{\infty}}\right)$ given by (3.1). Define the truncation function $\hat{\tau}_{+}: \mathbb{R} \rightarrow \mathbb{R}_{+}$by

$$
\hat{\tau}_{+}(s)= \begin{cases}0 & \text { if } s \leq 0 \\ s & \text { if } 0<s<\|\bar{u}\|_{\infty}, \\ \|\bar{u}\|_{\infty} & \text { if } s \geq\|\bar{u}\|_{\infty} .\end{cases}
$$

We introduce the functional $\hat{\varphi}_{+}: W_{0}^{1, p}(\Omega) \rightarrow \mathbb{R}$ as follows:

$$
\hat{\varphi}_{+}(u)=\frac{1}{p}\|D u\|_{p}^{p}-\int_{\Omega} \int_{0}^{u(x)} f\left(x, \hat{\tau}_{+}(s), \lambda\right) d s d x \text { for all } u \in W_{0}^{1, p}(\Omega) .
$$

Clearly, $\hat{\varphi}_{+}$is continuously differentiable, coercive and weakly lower semicontinuous. So there exists $u_{0}=u_{0}(\lambda) \in W_{0}^{1, p}(\Omega)$ such that

$$
\hat{\varphi}_{+}\left(u_{0}\right)=\inf \left\{\hat{\varphi}_{+}(u): u \in W_{0}^{1, p}(\Omega)\right\} .
$$

It turns out that $\hat{\varphi}_{+}^{\prime}\left(u_{0}\right)=0$, which is equivalent to

$$
-\Delta_{p} u_{0}=f\left(\cdot, \hat{\tau}_{+}\left(u_{0}(\cdot)\right), \lambda\right) \text { in } W^{-1, p^{\prime}}(\Omega) .
$$


By $\mathrm{H}(f)\left(\right.$ ii) it follows that $-\Delta_{p} u_{0} \in L^{\infty}(\Omega)$. From the nonlinear regularity theory (see [9], [11, 7, p.115-116]), we deduce $u_{0} \in C_{0}^{1}(\bar{\Omega})$. Using $-u_{0}^{-}=$ $-\max \left\{-u_{0}, 0\right\} \in W_{0}^{1, p}(\Omega)$ as a test function in (3.2) we obtain

$$
\left\|D u_{0}^{-}\right\|_{p}^{p}=\left\langle-\Delta_{p} u_{0},-u_{0}^{-}\right\rangle=-\int_{\Omega} f\left(x, \hat{\tau}_{+}\left(u_{0}(x)\right), \lambda\right) u_{0}^{-}(x) d x .
$$

Notice $\hat{\tau}_{+}\left(u_{0}(x)\right) \leq\|\bar{u}\|_{\infty}=\xi_{0}\|e\|_{\infty}<b$ and

$$
\int_{\Omega} f\left(x, \hat{\tau}_{+}\left(u_{0}(x)\right), \lambda\right) u_{0}^{-}(x) d x=0 .
$$

Hence we get $D u_{0}^{-}=0$, so $u_{0} \geq 0$.

By hypothesis, we can find $\mu=\mu(\lambda)>\lambda_{1}$ and $\delta=\delta(\lambda)>0$ such that

$$
\mu<\frac{f(x, s, \lambda)}{|s|^{p-2} s} \text { for a.a. } x \in \Omega \text { and all } 0<|s| \leq \delta .
$$

Choose $t>0$ with $t\left\|u_{1}\right\|_{\infty}<\min \left\{\delta,\|\bar{u}\|_{\infty}\right\}$. Then by (3.3) we have

$$
\hat{\varphi}_{+}\left(t u_{1}\right) \leq \frac{t^{p}}{p}\left(\lambda_{1}-\mu\right)<0
$$

which ensures that $\hat{\varphi}_{+}\left(u_{0}\right)<0=\hat{\varphi}_{+}(0)$, thus $u_{0} \neq 0$. Furthermore, because $f\left(x, \hat{\tau}_{+}\left(u_{0}(x)\right), \lambda\right) \geq 0$ a.e. on $\Omega($ cf. $\mathrm{H}(f)(\mathrm{iv}))$, from (3.2) we derive $-\Delta_{p} u_{0} \geq 0$ for a.e. on $\Omega$. The nonlinear strong maximum principle yields

$$
u_{0} \in \operatorname{int}\left(C_{0}^{1}(\bar{\Omega})_{+}\right)
$$

In addition, denoting $s^{+}=\max \{s, 0\}$ for $s \in \mathbb{R}$, we note from (3.2), $\mathrm{H}(f)$ (ii) and (3.1) that

$$
\begin{aligned}
\left\langle-\Delta_{p} u_{0}+\Delta_{p} \bar{u},\left(u_{0}-\bar{u}\right)^{+}\right\rangle & =\int_{\Omega}\left(f\left(x, \hat{\tau}_{+}\left(u_{0}(x)\right), \lambda\right)-\xi_{0}^{p-1}\right)\left(u_{0}-\bar{u}\right)^{+}(x) d x \\
& \leq \int_{\Omega}\left(\|a(\cdot, \lambda)\|_{\infty}+c\|\bar{u}\|_{\infty}^{r}-\xi_{0}^{p-1}\right)\left(u_{0}-\bar{u}\right)^{+} d x \leq 0,
\end{aligned}
$$

which shows $\left\|D\left(u_{0}-\bar{u}\right)^{+}\right\|_{p}^{p} \leq 0$, and thus $\left(u_{0}-\bar{u}\right)^{+}=0$. Consequently, we have $0<u_{0}(x) \leq \bar{u}(x)<b$ for all $x \in \Omega$ and from (3.2) we deduce that $u_{0}$ is a solution of problem (1.1). The fact that $u_{0}$ is with negative energy follows from $\hat{\varphi}_{+}\left(u_{0}\right)<0$.

Now set $\underline{u}=-\xi_{0} e \in-\operatorname{int}\left(C_{0}^{1}(\bar{\Omega})_{+}\right)$, with $\xi_{0} \in\left(0, \frac{b}{\|e\|_{\infty}}\right)$ given in (3.1), and introduce the truncation function $\hat{\tau}_{-}: \mathbb{R} \rightarrow \mathbb{R}_{-}$as

$$
\hat{\tau}_{-}(s)= \begin{cases}-\|\underline{u}\|_{\infty} & \text { if } s \leq-\|\underline{u}\|_{\infty}, \\ s & \text { if }-\|\underline{u}\|_{\infty}<s<0, \\ 0 & \text { if } s \geq 0 .\end{cases}
$$

Then we define the functional $\hat{\varphi}_{-}: W_{0}^{1, p}(\Omega) \rightarrow \mathbb{R}$ by

$$
\hat{\varphi}_{-}(u)=\frac{1}{p}\|D u\|_{p}^{p}-\int_{\Omega} \int_{0}^{u(x)} f\left(x, \hat{\tau}_{-}(s), \lambda\right) d s d x \text { for all } u \in W_{0}^{1, p}(\Omega) .
$$

Arguing with $\hat{\varphi}_{-}$in a similar way to the one for the functional $\hat{\varphi}_{+}$, we find a solution $v_{0}=v_{0}(\lambda) \in-\operatorname{int}\left(C_{0}^{1}(\bar{\Omega})_{+}\right)$to problem (1.1) such that $-b<\underline{u}(x) \leq v_{0}(x)$ on $\Omega$ and having negative energy. This completes the proof. 
MULTIPLE NONTRIVIAL SOLUTIONS FOR NONLINEAR EIGENVALUE PROBLEMS 3655

Remark 3.2. The functions $\bar{u}=\xi_{0} e$ and $\underline{u}=-\xi_{0} e$ constructed in the proof of Proposition 3.1 are an upper solution and a lower solution, respectively, for problem (1.1). In Proposition 3.1 the nonlinearity $f$ does not have a subcritical growth in the infinity. This is handled by employing a suitable truncation function argument used in [5].

\section{Proof of Theorem 1.1}

With the positive number $\lambda_{0}$ given by Proposition 3.1 fix $\lambda \in\left(0, \lambda_{0}\right)$. Let $u_{0} \in \operatorname{int}\left(C_{0}^{1}(\bar{\Omega})_{+}\right)$and $v_{0} \in-\operatorname{int}\left(C_{0}^{1}(\bar{\Omega})_{+}\right)$be the two solutions obtained in Proposition 3.1] We introduce on $\Omega \times \mathbb{R}$ the truncation functions

$$
\begin{gathered}
\tau_{+}(x, s)=\left\{\begin{array}{ll}
0 & \text { if } s \leq 0, \\
s & \text { if } 0<s<u_{0}(x),
\end{array} \tau_{-}(x, s)= \begin{cases}v_{0}(x) & \text { if } s \leq v_{0}(x), \\
s & \text { if } v_{0}(x)<s<0, \\
0 & \text { if } s \geq 0,\end{cases} \right. \\
u_{0}(x) \quad \text { if } s \geq u_{0}(x),
\end{gathered}
$$

For all $u \in W_{0}^{1, p}(\Omega)$ let us now define

$$
\begin{aligned}
& \varphi_{+}(u)=\frac{1}{p}\|D u\|_{p}^{p}-\int_{\Omega} \int_{0}^{u(x)} f\left(x, \tau_{+}(x, s), \lambda\right) d s d x, \\
& \varphi_{-}(u)=\frac{1}{p}\|D u\|_{p}^{p}-\int_{\Omega} \int_{0}^{u(x)} f\left(x, \tau_{-}(x, s), \lambda\right) d s d x, \\
& \varphi_{0}(u)=\frac{1}{p}\|D u\|_{p}^{p}-\int_{\Omega} \int_{0}^{u(x)} f\left(x, \tau_{0}(x, s), \lambda\right) d s d x,
\end{aligned}
$$

and notice that $\varphi_{+}, \varphi_{-}, \varphi_{0} \in C^{1}\left(W_{0}^{1, p}(\Omega)\right)$.

It readily follows that $u_{0}$ is a critical point of $\varphi_{+}$because $u_{0}$ is a solution of problem (1.1). Let us show that

$$
v \text { is a critical point of } \varphi_{+} \Longrightarrow 0 \leq v(x) \leq u_{0}(x) \text { for a.a. } x \in \Omega \text {. }
$$

To this end, let $v$ be a critical point of $\varphi_{+}$. We note that

$$
\left\langle\Delta_{p} u_{0}-\Delta_{p} v,\left(v-u_{0}\right)^{+}\right\rangle=\int_{\Omega}\left(f\left(x, \tau_{+}(x, v(x)), \lambda\right)-f\left(x, u_{0}(x), \lambda\right)\right)\left(v-u_{0}\right)^{+} d x=0,
$$

which implies $v \leq u_{0}$. Similarly, we check that $v \geq 0$, which leads to (4.1). On the basis of (4.1) we see that every critical point $v$ of $\varphi_{+}$is a solution of (1.1).

We also observe that the function $\varphi_{+}$is coercive, weakly lower semicontinuous, so $\varphi_{+}$has a global minimizer $z_{0} \in W_{0}^{1, p}(\Omega)$. Furthermore, using (3.3), it turns out

$$
\varphi_{+}\left(z_{0}\right)=\inf _{W_{0}^{1, p}(\Omega)} \varphi_{+}<0,
$$

thus $z_{0} \neq 0$. If $z_{0} \neq u_{0}$, then by (4.1) the desired conclusion follows with $w_{0}=z_{0}$. So we may assume that $z_{0}=u_{0}$, and it is the unique global minimizer of $\varphi_{+}$(see (4.1)). On the other hand, according to (3.4) we find a neighborhood $V$ of $u_{0}$ in the space $C_{0}^{1}(\bar{\Omega})$ such that $V \subset C_{0}^{1}(\bar{\Omega})_{+}$, thus $\varphi_{0}=\varphi_{+}$on $V$. This guarantees that $u_{0}$ is a local minimizer of $\varphi_{0}$ on $C_{0}^{1}(\bar{\Omega})$. Then it follows that $u_{0}$ is a local minimizer of $\varphi_{0}$ in the space $W_{0}^{1, p}(\Omega)$ (see [7, p. 655-656]). By the same reasoning as above for 
$\varphi_{+}$, we may admit that $v_{0}$ is the unique global minimizer of $\varphi_{-}$. Proceeding as in the case of $u_{0}$, we establish that $v_{0}$ is a local minimizer of $\varphi_{0}$ on $W_{0}^{1, p}(\Omega)$.

As before in (4.1) we verify that every critical point of $\varphi_{0}$ belongs to the set $\left\{u \in W_{0}^{1, p}(\Omega): v_{0}(x) \leq u(x) \leq u_{0}(x)\right.$ a.e. $\left.x \in \Omega\right\}$. This implies that every critical point of $\varphi_{0}$ is a solution to problem (1.1). The functional $\varphi_{0}$ is coercive, weakly lower semicontinuous, so it is bounded from below. In addition, one has $\inf _{W_{0}^{1, p}(\Omega)} \varphi_{0}<0$. Thus $\varphi_{0}$ has a global minimizer $y_{0} \in W_{0}^{1, p}(\Omega)$ with $y_{0} \neq 0$. If $y_{0} \neq u_{0}$ and $y_{0} \neq v_{0}$, then the conclusion in the theorem is obtained by taking $w_{0}=y_{0}$. It remains to consider the cases $y_{0}=u_{0}$ or $y_{0}=v_{0}$. To make a choice, suppose $y_{0}=u_{0}$. We may also admit that $v_{0}$ is a strict local minimizer of $\varphi_{0}$ since on the contrary we are done. Consequently, we can find $\rho \in\left(0,\left\|u_{0}-v_{0}\right\|\right)$ such that

$$
\varphi_{0}\left(u_{0}\right) \leq \varphi_{0}\left(v_{0}\right)<\inf \left\{\varphi_{0}(u): u \in \partial B_{\rho}\left(v_{0}\right)\right\},
$$

where $\partial B_{\rho}\left(v_{0}\right)=\left\{u \in W_{0}^{1, p}(\Omega):\left\|u-v_{0}\right\|=\rho\right\}$. Relation (4.2) in conjunction with the $\mathrm{PS}_{c}$-condition for $\varphi_{0}$ at any $c \in \mathbb{R}$ (due to its coercivity) enables us to apply the mountain pass theorem to $\varphi_{0}$ (see, e.g., [10]) and get $w_{0} \in W_{0}^{1, p}(\Omega)$ satisfying $\varphi_{0}^{\prime}\left(w_{0}\right)=0$ and

$$
\inf \left\{\varphi_{0}(u): u \in \partial B_{\rho}\left(v_{0}\right)\right\} \leq \varphi_{0}\left(w_{0}\right)=\inf _{\gamma \in \Gamma} \max _{t \in[-1,1]} \varphi_{0}(\gamma(t))
$$

where

$$
\Gamma=\left\{\gamma \in C\left([-1,1], W_{0}^{1, p}(\Omega)\right): \gamma(-1)=v_{0}, \gamma(1)=u_{0}\right\} .
$$

We infer from (4.2) and (4.3) that $w_{0} \neq u_{0}$ and $w_{0} \neq v_{0}$. Next let us prove that

$$
\varphi_{0}\left(w_{0}\right)<0 \text {. }
$$

Towards this, in view of (4.3), it suffices to produce a path $\hat{\gamma} \in \Gamma$ such that

$$
\varphi_{0}(\hat{\gamma}(t))<0 \text { for all } t \in[-1,1] \text {. }
$$

Let $S=W_{0}^{1, p}(\Omega) \cap \partial B_{1}^{L^{p}(\Omega)}$ and $S_{C}=S \cap C_{0}^{1}(\bar{\Omega})$ be endowed with the topologies induced by $W_{0}^{1, p}(\Omega)$ and $C_{0}^{1}(\bar{\Omega})$, respectively. We set

$$
\Gamma_{0, C}=\left\{\gamma \in C\left([-1,1], S_{C}\right): \gamma(-1)=-u_{1}, \gamma(1)=u_{1}\right\}
$$

In view of assumption $\mathrm{H}(f)$ (iii), fix numbers $\mu>\lambda_{2}$ and $\delta>0$ satisfying (3.3), and let $\rho_{0} \in\left(0, \mu-\lambda_{2}\right)$. By (2.2) there exists $\gamma \in \Gamma_{0}$ such that

$$
\max _{t \in[-1,1]}\|D \gamma(t)\|_{p}^{p}<\lambda_{2}+\frac{\rho_{0}}{2} .
$$

Choose some $r$ with $0<r \leq\left(\lambda_{2}+\rho_{0}\right)^{\frac{1}{p}}-\left(\lambda_{2}+\frac{\rho_{0}}{2}\right)^{\frac{1}{p}}$. The density of $S_{C}$ in $S$ implies that $\Gamma_{0, C}$ is dense in $\Gamma_{0}$, so there is $\gamma_{0} \in \Gamma_{0, C}$ satisfying

$$
\max _{t \in[-1,1]}\left\|D \gamma(t)-D \gamma_{0}(t)\right\|_{p}<r .
$$

This ensures

$$
\max _{t \in[-1,1]}\left\|D \gamma_{0}(t)\right\|_{p}^{p}<\lambda_{2}+\rho_{0}
$$

Thanks to the boundedness of $\gamma_{0}([-1,1])(\bar{\Omega})$ in $\mathbb{R}$ there exists $\varepsilon>0$ such that

$$
\varepsilon|u(x)| \leq \delta \text { for all } x \in \Omega \text { and all } u \in \gamma_{0}([-1,1]) \text {. }
$$


Since $u_{0},-v_{0} \in \operatorname{int}\left(C_{0}^{1}(\bar{\Omega})_{+}\right)$, for every $u \in \gamma_{0}([-1,1])$, we can find arbitrarily large positive numbers $h_{u}$ and $j_{u}$ satisfying $h_{u} u_{0}-u,-j_{u} v_{0}+u \in \operatorname{int}\left(C_{0}^{1}(\bar{\Omega})_{+}\right)$. Then there exists a neighborhood $V_{u}$ of $u$ in $C_{0}^{1}(\bar{\Omega})$ such that

$$
h_{u} u_{0}-v \in \operatorname{int}\left(C_{0}^{1}(\bar{\Omega})_{+}\right) \text {and }-j_{u} v_{0}+v \in \operatorname{int}\left(C_{0}^{1}(\bar{\Omega})_{+}\right) \text {for all } v \in V_{u} .
$$

Using the compactness of $\gamma_{0}([-1,1])$ in $C_{0}^{1}(\bar{\Omega})$ a number $\zeta>0$ can be determined to fulfill

$$
v_{0}(x) \leq \varepsilon u(x) \leq u_{0}(x) \text { for all } x \in \Omega \text { and all } u \in \gamma_{0}([-1,1])
$$

provided $\varepsilon \in(0, \zeta)$. By (4.6), (4.8), (4.7), (3.3) and the fact that $\gamma_{0}([-1,1]) \subset$ $\partial B_{1}^{L^{p}(\Omega)}$ we obtain for the continuous path $\varepsilon \gamma_{0}$ joining $-\varepsilon u_{1}, \varepsilon u_{1}$ that

$$
\begin{aligned}
\varphi_{0}\left(\varepsilon \gamma_{0}(t)\right) & =\frac{\varepsilon^{p}}{p}\left\|D \gamma_{0}(t)\right\|_{p}^{p}-\int_{\Omega} \int_{0}^{\varepsilon \gamma_{0}(t)(x)} f\left(x, \tau_{0}(x, s), \lambda\right) d s d x \\
& \leq \frac{\varepsilon^{p}}{p}\left(\lambda_{2}+\rho_{0}-\mu\right)<0 \quad \text { for all } t \in[-1,1] .
\end{aligned}
$$

Let us denote $c_{+}=c_{+}(\lambda)=\varphi_{+}\left(\varepsilon u_{1}\right)$ and $m_{+}=m_{+}(\lambda)=\varphi_{+}\left(u_{0}\right)$. Since $u_{0}$ is the unique global minimizer of $\varphi_{+}$, we have that $m_{+}<c_{+}$. Taking into account (4.1) and that $c_{+}=\varphi_{0}\left(\varepsilon u_{1}\right)<0$, we may assume that $K_{c_{+}}\left(\varphi_{+}\right)=\emptyset$ and $\varphi_{+}$has no critical values in the open interval $\left(m_{+}, c_{+}\right)$(for, otherwise, a third solution of (1.1) is found with the properties in the statement of the theorem). Moreover, $\varphi_{+}^{m_{+}}=$ $\left\{u_{0}\right\}$ and $\varphi_{+}$satisfies the $\mathrm{PS}_{c}$-condition at any level $c \in \mathbb{R}$ because it is coercive. Therefore all the assumptions in Lemma 2.1 are verified with $a=m_{+}$and $b=c_{+}$ for the functional $\varphi_{+}$. Lemma 2.1 yields a homotopy $\eta \in C\left([0,1] \times \varphi_{+}^{c_{+}}, \varphi_{+}^{c_{+}}\right)$such that $\eta(0, u)=u$ and $\eta(1, u)=u_{0}$ for all $u \in \varphi_{+}^{c_{+}}$, as well as $\varphi_{+}(\eta(t, u)) \leq \varphi_{+}(u)$ whenever $t \in[0,1]$ and $u \in \varphi_{+}^{c_{+}}$. Defining $\gamma_{+}:[0,1] \rightarrow W_{0}^{1, p}(\Omega)$ by $\gamma_{+}(t)=\eta\left(t, \varepsilon u_{1}\right)$ for all $t \in[0,1]$, it is seen that $\gamma_{+}$is a continuous path in $W_{0}^{1, p}(\Omega)$ joining $\varepsilon u_{1}$ and $u_{0}$. We claim that

$$
\varphi_{0}\left(\gamma_{+}(t)\right) \leq \varphi_{+}\left(\gamma_{+}(t)\right) \text { for all } t \in[0,1] .
$$

If $\gamma_{+}(t)(x)<0$, then, in view of $\mathrm{H}(f)$ (iv), we have for all $s \in\left[\gamma_{+}(t)(x), 0\right]$ that $f\left(x, \tau_{0}(x, s), \lambda\right) \leq 0=f\left(x, \tau_{+}(x, s), \lambda\right)$. If $\gamma_{+}(t)(x) \geq 0$, then for all $s \in\left[0, \gamma_{+}(t)(x)\right]$ there holds $f\left(x, \tau_{0}(x, s), \lambda\right)=f\left(x, \tau_{+}(x, s), \lambda\right)$, so the claim follows from the expressions of $\varphi_{0}$ and $\varphi_{+}$. On the basis of (4.9) and the properties of the deformation $\eta$ we get

$$
\varphi_{0}\left(\gamma_{+}(t)\right) \leq \varphi_{+}\left(\eta\left(t, \varepsilon u_{1}\right)\right) \leq \varphi_{+}\left(\varepsilon u_{1}\right)<0 \text { for all } t \in[0,1] .
$$

Similarly, applying Lemma 2.1 for the functional $\varphi_{-}$, we construct a continuous path $\gamma_{-}:[0,1] \rightarrow W_{0}^{1, p}(\Omega)$ joining $v_{0}$ and $-\varepsilon u_{1}$ such that $\varphi_{0}\left(\gamma_{-}(t)\right)<0$ for all $t \in[0,1]$.

Piecing together the paths $\gamma_{-}, \varepsilon \gamma_{0}$ and $\gamma_{+}$, we produce a continuous curve $\hat{\gamma} \in \Gamma$ joining $v_{0}$ and $u_{0}$ that satisfies (4.5). Hence (4.4) holds, which implies $w_{0} \neq 0$. Recalling that the critical points of $\varphi_{0}$ are in the order interval $\left\{u \in W_{0}^{1, p}(\Omega)\right.$ : $v_{0}(x) \leq u(x) \leq u_{0}(x)$ a.e. $\left.x \in \Omega\right\}$ we derive that $w_{0}$ is a nontrivial solution of (1.1) distinct from $v_{0}$ and $u_{0}$, with $v_{0}(x) \leq w_{0}(x) \leq u_{0}(x)$ for a.a. $x \in \Omega$ and negative energy (see (4.4) ). By the nonlinear regularity theory we have that $w_{0} \in C_{0}^{1}(\bar{\Omega})$ which completes the proof. 


\section{ACKNOWLEDGMENT}

The authors express their gratitude to the referee for his valuable remarks.

\section{REFERENCES}

1. A. Ambrosetti, H. Brezis, and G. Cerami, Combined effects of concave and convex nonlinearities in some elliptic problems, J. Funct. Anal. 122 (1994), 519-543. MR1276168 (95g:35059)

2. A. Anane and N. Tsouli, On the second eigenvalue of the p-Laplacian. In: Nonlinear Partial Differential Equations (eds. A. Benikrane - J. P. Gossez), Pitman Research Notes in Math. 343, Longman, Harlow, 1996, 1-9. MR1417265 (97k:35190)

3. S. Carl and K. Perera, Sign-changing and multiple solutions for the p-Laplacian, Abstr. Appl. Anal. 7 (2002), 613-625. MR1950611(2004a:35061)

4. M. Cuesta, D. de Figueiredo, and J.-P. Gossez, The beginning of the Fučik spectrum for the p-Laplacian, J. Differential Equations 159 (1999), 212-238. MR.1726923 (2001f:35308)

5. J. Deuel and P. Hess, A criterion for the existence of solutions of non-linear elliptic boundary value problems, Proc. Roy. Soc. Edinburgh Sect. A 74 (1974/75), 49-54. MR0440191 $(55: 13070)$

6. J. Garcia Azorero, I. Peral, and J. Manfredi, Sobolev versus Hölder local minimizers and global multiplicity for some quasilinear elliptic equations, Commun. Contemp. Math. 2 (2000), 385404. MR1776988 (2001k:35062)

7. L. Gasiński and N. S. Papageorgiou, Nonsmooth Critical Point Theory and Nonlinear Boundary Value Problems. Chapman \& Hall/CRC, Boca Raton, FL, 2005. MR2092433 (2006f:58013)

8. Z. Jin, Multiple solutions for a class of semilinear elliptic equations, Proc. Amer. Math. Soc. 125 (1997), 3659-3667. MR 1443158 (98h:35078)

9. G. M. Lieberman, Boundary regularity for solutions of degenerate elliptic equations, Nonlinear Anal. 12 (1988), 1203-1219. MR.969499 (90a:35098)

10. P. H. Rabinowitz, Minimax Methods in Critical Point Theory with Applications to Differential Equations, CBMS Regional Conference Series in Mathematics, vol. 65, American Mathematical Society, Providence, RI, 1986. MR845785 (87j:58024)

11. P. Tolksdorf, Regularity for a more general class of quasilinear elliptic equations, J. Differential Equations 51 (1984), 126-150. MR727034 (85g:35047)

12. J. L. Vázquez, A strong maximum principle for some quasilinear elliptic equations, Appl. Math. Optimization 12 (1984), 191-202. MR768629 (86m:35018)

Département de Mathématiques, Université de Perpignan, 66860 Perpignan, France E-mail address: motreanu@univ-perp.fr

Département de Mathématiques, Université de Perpignan, 66860 Perpignan, France

E-mail address: viorica@univ-perp.fr

Department of Mathematics, National Technical University, Athens 15780, Greece

E-mail address: npapg@math.ntua.gr 